\title{
Macular edema resolution assessment with implantable dexamethasone in diabetic retinopathy (MERIT): a pilot study
}

This article was published in the following Dove Press journal:

Clinical Ophthalmology

Jay Chhablani

Mahima Jhingan

Abhilash Goud

Kiran Kumar Vupparaboina

Taraprasad Das

Smt Kanuri Santhamma Retina

Vitreous Centre, LV Prasad Eye

Institute, Hyderabad, Telangana, India
Correspondence: Jay Chhablani Smt Kanuri Santhamma Retina Vitreous Centre, LV Prasad Eye Institute, Kallam Anji Reddy Campus, 2 LV Prasad Marg Banjara Hills, Hyderabad 500034,

Telangana, India

Tel +9l $40306 \mid 2603$

Fax +9| $402354827 \mid$

Email jay.chhablani@gmail.com
Purpose: This study aimed to evaluate the effect of dexamethasone implantation on the hard exudates (HEX) in patients with diabetic macular edema (DME).

Study design: This was a nonrandomized open-label single-center prospective trial.

Methods: This study included 15 eyes of 11 subjects with DME. Key inclusion criteria were naïve eyes with DME with HEX within $3 \mathrm{~mm}$ of fovea with center-involving DME; central macular thickness $(\mathrm{CMT})>250 \mu \mathrm{m}$ at baseline; best-corrected visual acuity (BCVA) between $20 / 400$ and 20/40. Key exclusion criteria were previous intraocular surgery and history of panretinal photocoagulation (PRP) in past 4 months. The primary outcome measure was change in total HEX area at the macula (in $\mathrm{mm}^{2}$ ) measured by semiautomated algorithm. Secondary outcome measures were change in visual acuity, low-contrast visual acuity (LCVA), retinal sensitivity (RS) on macular microperimetry, and CMT.

Results: The total HEX area reduced from $1.5 \mathrm{~mm}^{2}\left( \pm 1.46 \mathrm{~mm}^{2}\right)$ at baseline to $0.89 \mathrm{~mm}^{2}$ $\left( \pm 1.062 \mathrm{~mm}^{2}\right)$ at the final visit $(p=0.185)$. The CMT improved significantly $(p=0.03)$ from $488.67 \mu \mathrm{m}( \pm 240.66 \mu \mathrm{m})$ to $326.93 \mu \mathrm{m}( \pm 135.84 \mu \mathrm{m})$ at the final visit. Mean BCVA remained stable ( $p=0.95)(50.93 \pm 16.65$ at baseline and 50.6 \pm 18.95 at final visit). The mean LCVA and RS showed insignificant improvement ( $p=0.31$ and $p=0.28$, respectively).

Conclusion: Our pilot study demonstrated an improving trend in reduction of total HEX area and other anatomical outcomes, with limited functional outcomes. Larger randomized studies with a larger sample size with a control group are warranted to establish management protocols for DME with significant subfoveal HEX.

Keywords: dexamethasone implant, Ozurdex, hard exudates, diabetic macular edema

\section{Introduction}

Diabetic macular edema (DME) constitutes a significant burden owing to the prolonged and varied response to treatment. DME results mainly from hyperpermeability of retinal vessels and microaneurysms associated with deposition of lipoproteins, clinically manifesting as hard exudates (HEX), mostly deposited in the posterior pole, often signifying the chronicity of the edema. ${ }^{1}$ Subfoveal migration of HEX could be a challenging situation, with very poor visual outcome due to outer retinal structure damage and may lead to subretinal fibrosis.

Various approaches, including laser photocoagulation, anti-vascular endothelial growth factor (VEGF) therapy, surgical removal, and intravitreal triamcinolone have been used to manage extensive HEX..$^{2-7}$ Each of the treatment modalities has its challenges, such as the associated complications and limitations. Anti-VEGF therapy has shown promising results in terms of both anatomical and visual outcomes and 
has rapidly become the standard of care after pivotal trials such as the RISE and RIDE. ${ }^{8}$ However, rapid reduction of DME is associated with an increase in intraretinal exudates, along with a shift to a more outer retinal location. ${ }^{9}$ On the other hand, the RIDE and RISE trials have demonstrated that the area of HEX in eyes with DME did not affect visual prognosis and that HEX did not increase in ranibizumabtreated eyes. ${ }^{10}$

Considering that DME could primarily be an inflammatory pathological process, intravitreal steroids have also been used, and these have shown promising results in the reduction of HEX and edema over a short term of follow-up. ${ }^{4-6,11}$ Intravitreal triamcinolone is associated with various possible complications such as endophthalmitis and intractable glaucoma. ${ }^{12}$ The 2-year results of the Bevacizumab or Dexamethasone Implants for DME (BEVORDEX) study showed a reduction of HEX in patients with DME with both intravitreal dexamethasone and bevacizumab, although the reduction of HEX in center-involving DME $(<500 \mu \mathrm{m})$ was statistically significant with dexamethasone only. ${ }^{13}$

Many large-scale clinical trials such as protocol $\mathrm{T}^{14} \mathrm{did}$ not include eyes with extensive exudates involving foveal area; therefore, clinicians lack any established protocol to treat such cases. We designed a pilot study to document the effect of continuous release of dexamethasone (using an implantable device) on the HEX at fovea (primary objective) in patients with DME and to quantify the same. The secondary objective was to study the functional impact of implantable dexamethasone on the contrast sensitivity and macular function.

\section{Methods}

A nonrandomized open-label single-center prospective controlled trial was conducted on 15 eyes of 11 subjects with a diagnosis of DME who presented to the LV Prasad Eye Institute between April 2015 and February 2016. Written informed consent was obtained from all subjects. The institutional review board of Hyderabad Eye Research Foundation approved the study (LEC12-14-109), and all the procedures adhered to the tenets of the Declaration of Helsinki. The chart review followed the previously set out guidelines, described in earlier publications. ${ }^{15}$

Key inclusion criteria for the study were as follows: 1) naïve eyes with DME with HEX within $3 \mathrm{~mm}$ of fovea with center-involving DME; 2) diagnosis of diabetes mellitus (type 1 or type 2 ); 3) central foveal thickness $>250 \mu \mathrm{m}$ at baseline; and 4) best-corrected visual acuity (BCVA) between 20/400 and 20/40. The exclusion criteria included the following: 1) any other associated ocular condition; 2) macular edema due to any other condition; 3) presence of conditions such as macular ischemia, vitreomacular traction, foveal atrophy, or pigment abnormalities; 4) substantial cataract likely to decrease visual acuity by $\geq 3$ lines; 5) history of treatment for DME at any time; 6) history of panretinal (scatter) photocoagulation (PRP) within 4 months prior to enrollment; 7) history of major ocular surgery (including vitrectomy, cataract extraction, scleral buckle, any intraocular surgery, and so on) within the previous 4 months or anticipated within the next 6 months following enrollment; 8) history of yttrium aluminum garnet (YAG) capsulotomy performed within 2 months prior to enrollment; 9) aphakia; 10) intraocular pressure (IOP) $\geq 25 \mathrm{mmHg}$; 11) history of open-angle glaucoma; 12) history of steroid-induced IOP elevation that required IOP-lowering treatment; and 13) examination evidence of external ocular infection, including conjunctivitis, chalazion, or significant blepharitis.

Data collected at the initial visit included demographics, BCVA using the Early Treatment Diabetic Retinopathy Study (ETDRS) visual acuity, low-contrast visual acuity (LCVA) using COMPLog, color fundus photographs (7 fields), infrared images ( 7 fields), autofluorescence images ( 7 fields), optical coherence tomography (OCT) with central macular thickness (CMT), fundus fluorescein angiography (FFA), and microperimetry.

A comprehensive ocular examination was performed in all cases. Ocular investigations included OCT and FFA. FFA and fundus photography were performed using FF450 plus Fundus Camera (Carl Zeiss, Jena, Germany) with VISUPAC. Lens grading was performed as per standard Emery and Little classification. ${ }^{16}$ Patients were not treated aggressively with lipid-lowering medications, other than their regular medications.

\section{Color fundus photographs}

Color fundus photographs $\left(50^{\circ}\right.$ and $\left.30^{\circ}\right)$ were captured in 7 fields with a mydriatic fundus camera (Zeiss FF450; Carl Zeiss Meditec).

\section{FFA study}

FFA was performed using fluorescein sodium 20\%, and imaging was performed using FF450 plus Fundus Camera (Carl Zeiss Meditec) with VISUPAC in all visits.

\section{Spectral domain OCT (SD-OCT)}

The SD-OCT scans were obtained using Cirrus HD-OCT (Carl Zeiss Meditec) after dilatation of the pupil with $0.8 \%$ 
tropicamide and 5\% phenylephrine eye drops at every visit. The scanning protocol included high-definition (HD) 5-line raster, HD single-line raster, enhanced depth imaging, and macular cube. Central retinal thickness (CRT) was determined automatically and analyzed by OCT software, by generating images using the Macular Cube $512 \times 128$ scan over a $6 \times 6 \mathrm{~mm}$ area, the cube being composed of 128 horizontal examination lines of $512 \mathrm{~A}$-scans each. The CRT was obtained from the $1 \mathrm{~mm}$ CRT area as described in the ETDRS fields corresponding to the CMT.

\section{Microperimetry}

Microperimetry was performed using the microperimeter (macular integrity assessment $\left[\mathrm{MAIA}^{\mathrm{TM}}\right.$; ; CenterVue, Padova, Italy). Pupils of all patients were dilated before examination. Goldmann III stimuli and a 4-2-1 staircase strategy were used, and a test grid with 37 stimulus locations covering an area of $10^{\circ}$ was applied. The stimuli were projected on a white background with black illumination set to $1.27 \mathrm{~cd} / \mathrm{m}^{2}$ and a stimulus presentation time of $200 \mathrm{~ms}$. The perimetric strategy of the microperimeter starts at an initially defined threshold level for each stimulus. A 4-2-1 staircase strategy is then carried out, and the weakest recognized value is documented as the retinal sensitivity (RS) at each tested site. Mean differential light sensitivity, in decibels (dB), of all test locations was analyzed for the study. For assessment of fixation, the fundus movements were tracked during examination while the patient gazed at the fixation target. The auto tracking system calculated the horizontal and vertical shifts relative to a reference frame and drew a map of the patient's eye movements during the examination. The recorded fixation points were classified into 3 categories for fixation analysis - stable, relatively unstable, and unstable. Fixation was defined as follows: stable if $>75 \%$ of the fixation points were inside the $2^{\circ}$-diameter circle; relatively unstable if $<75 \%$ were inside the $2^{\circ}$-diameter circle, but $>75 \%$ were inside the $4^{\circ}$-diameter circle; and unstable if $<75 \%$ of the fixation sites were inside the $4^{\circ}$-diameter circle. Significant improvement or worsening in microperimetry was defined as a change in sensitivity of $\geq 1 \mathrm{~dB}$, a change in stability of fixation, or both.

\section{Measurements of HEX by a semiautomated algorithm}

We used the semiautomated algorithm for HEX measurements; this algorithm uses a 2-pronged methodology, involved performing top-hat filtering, second-order statistical filtering, and thresholding of the color fundus images.
In brief, a 2-pronged approach was adopted to detect both bright and weak/faint HEX. In Step 1, to detect bright HEX, top-hat ${ }^{17}$ filtering was first used on the gray scale image of the color fundus photograph using a structuring element (disk, where the radius of the disk is considered to be $1 / 4$ the width of the image) to make background illumination more uniform. Subsequently, adaptive histogram equalization ${ }^{17}$ was performed to further enhance the exudates from the background. Then, the enhanced image was binarized using an empirically determined threshold (0.37) for segmenting the exudates from the background, in which the brighter pixels indicate the exudates. However, in addition to the exudates, some outliers also were obtained due to reflections from the optic disk and retinal vessels in the nerve fiber layer.

Next, in Step 2, we proceed to detect the weaker exudates that may not have been detected in Step 1. Here, only the green plane of the color fundus image is considered because weak exudates are better contrasted in the green plane than in the grayscale image or the red and blue planes. Then, similar operations that were used in Step 1 were subsequently performed but with different thresholds. Specifically, the radius of the structuring element for performing top-hat filtering is decreased to one quarter of the width of the image, and the threshold for binarization is empirically determined as 0.25 for images obtained after the respective operations. Step 2 also resulted in a few outliers due to the same reasons mentioned earlier in Step 1.

Now, the results of Step 1 and Step 2 are combined, and the outliers are removed manually using a rectangular selection box. Finally, the total area of the detected HEX is measured to facilitate monitoring of the treatment response.

Color fundus photographs were analyzed using this algorithm by a single observer. Intraobserver reproducibility was evaluated using the first 15 images and was found to correlate well, with an intraclass correlation coefficient of 0.94 .

\section{Intravitreal injection of the dexamethasone implant}

All individuals who met the study criteria underwent intravitreal injection of the dexamethasone $(0.7 \mathrm{mg})$ implant into the eye following all aseptic precautions in a sterile environment. After topical anesthesia and Betadine ${ }^{\circledR}$ (Mhs Pharmaceuticals Private Limited, Hyderabad, India) 10\% preparation of the eye, the implant was injected in the inferotemporal quadrant. When 2 eyes of the same individual fit the study criteria, the 2 eyes were injected at an interval of 3 days. No postinjection prophylaxis or patching was recommended. Injections were performed at baseline and at Month 4 . 


\section{Study visits}

All patients were examined at baseline and at Days 60, 120, 180, and 240. Comprehensive examination, BCVA, LCVA, fundus photography, SD-OCT, and microperimetry were done at all visits. FFA was performed at baseline, Day 120, and Day 240. All individuals were advised to get the IOP measured locally at 2 weeks after the intravitreal dexamethasone implant injection, to rule out any inadvertent steroid-related rise in IOP.

\section{Outcome measures}

\section{Primary outcome measure}

The primary outcome of interest was the change in total area of HEX at the macula (in $\mathrm{mm}^{2}$ ), measured by a semiautomated algorithm.

\section{Secondary outcome measures}

The secondary outcome measures were changes in visual acuity, LCVA, RS on macular microperimetry, and central subfield thickness on SD-OCT.

\section{Statistical analysis}

Statistical analysis was performed using SPSS software (version 16.0; SPSS, Chicago, IL, USA), with special emphasis on the aforementioned information. Wilcoxon signed-rank test was used for statistical analysis of various outcome measures. The follow-up rate was $87 \%$, and therefore, last observation carried forward (LOCF) analysis was performed. Values of $p<0.05$ were considered statistically significant.

\section{Results}

Fifteen eyes of 11 patients, 6 males and 5 females, were included in the study. Mean age was $54.4 \pm 8.1$ years. All patients had type 2 diabetes mellitus. Three patients were on antihypertensive medications, and 2 patients were on lipid-lowering medications. None of the patients were smokers. All eyes, but 1, were phakic. Phakic eyes had grade I nuclear sclerosis in 5 eyes and grade 2 in 3 eyes; the remaining 6 eyes had clear crystalline lens. A representative case of the phakic eye is shown in Figure 1.

\section{Anatomical outcome}

The total area of HEX at the macula was $1.5 \mathrm{~mm}^{2}\left( \pm 1.46 \mathrm{~mm}^{2}\right)$ at presentation. This reduced to $0.89 \mathrm{~mm}^{2}\left( \pm 1.062 \mathrm{~mm}^{2}\right)$ at the final visit. This was a clinically significant decrease over the short duration even though statistically insignificant $(p=0.185)$.
The CMT of the patients in our study improved significantly $(p=0.03)$ from a baseline of $488.67 \mu \mathrm{m}( \pm 240.66 \mu \mathrm{m})$ to $326.93 \mu \mathrm{m}( \pm 135.84 \mu \mathrm{m})$.

\section{Visual outcome}

Mean BCVA at presentation was $50.93( \pm 16.65)$ ETDRS letters. At the 8-month follow-up, BCVA remained stable at $50.6( \pm 18.95)$ ETDRS letters; this was statistically not significant $(p=0.95)$. The mean LCVA at presentation was 1.15 ( \pm 0.39 ) (approximate Snellen's equivalent of 20/300), which improved to $0.97( \pm 0.43)$ (approximate Snellen's equivalent of 20/200) at the final visit; this was statistically not significant $(p=0.31)$.

\section{Functional outcome}

The average threshold microperimetry values improved from $6.99 \mathrm{~dB}( \pm 8.897 \mathrm{~dB})$ at presentation to $11.91 \mathrm{~dB}( \pm 7.64 \mathrm{~dB})$ at final follow-up. This showed a trend toward an improvement in the macular function over time despite the results appearing statistically insignificant $(p=0.28)$.

\section{Cataract progression}

Among the phakic eyes, 1 eye with grade I nuclear sclerosis at baseline had posterior subcapsular cataract and 4 eyes with clear crystalline lens at baseline had early nuclear sclerosis at the final visit.

\section{Change in IOP}

Mean IOP at baseline was $13.2 \pm 2.59 \mathrm{mmHg}$, which remained stable till the final visit at $14.46 \pm 3.46 \mathrm{mmHg}(p=0.26)$. One patient had an increase in IOP (26 mmHg) at Month 2, which was controlled using antiglaucoma medication till the final visit.

\section{Diabetic retinopathy grading}

At baseline, 14 eyes had moderate nonproliferative diabetic retinopathy (NPDR) and 1 eye had stable lasered proliferative diabetic retinopathy (PDR; lasering was done 9 months before the enrollment). None of the eyes had change in their diabetic retinopathy status till the final visit.

Table 1 shows a short summary of the results.

\section{Discussion}

Our study showed a trend toward decrease in total surface area of HEX at the macula, along with an increase in RS, following dexamethasone implantation. Though these results were not significant, they bode well for long-term follow-up of patients on these implants. A trend toward decreasing HEX in DME has been described with both anti-VEGF injections ${ }^{10}$ 

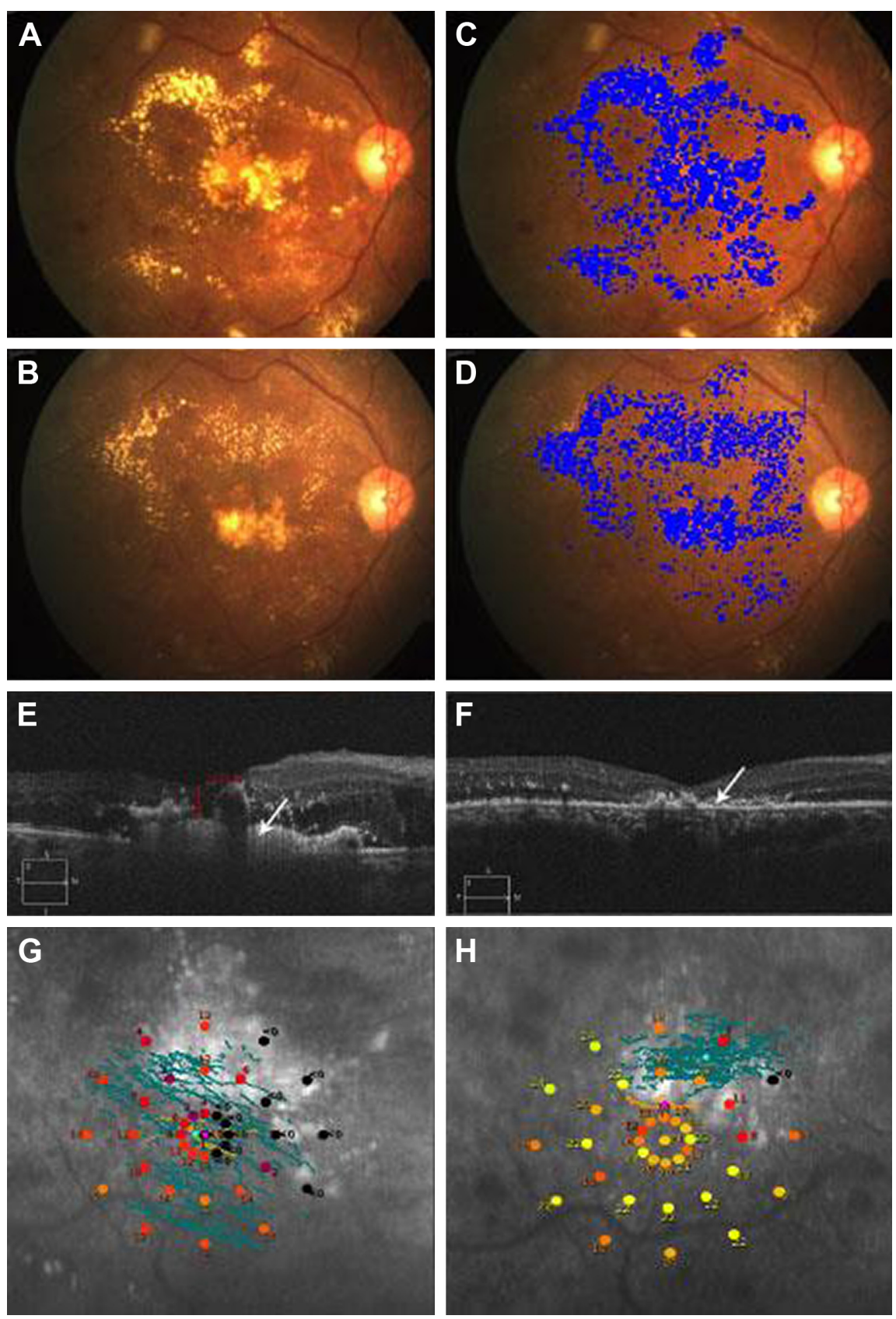

Figure I Representative case of phakic eye.

Notes: (A) A 56-year-old male presented with moderate NPDR and center-involving macular edema with plenty of HEX. He underwent 2 intravitreal dexamethasone implantations at baseline and at Month 4. (B) His visual acuity improved from 20/200 to 20/100 at Month 8, with reduction in HEX. Quantitative analysis of HEX showed a reduction from (C) $1.9 \mathrm{~mm}^{2}$ to (D) $1.75 \mathrm{~mm}^{2}$. Spectral domain optical coherence tomography showed a reduction in subfoveal HEX (arrow) and reduction in central macular thickness as can be seen at $(\mathbf{E})$ baseline and $(\mathbf{F})$ Month 8. Retinal sensitivity map shows improvement from $(\mathbf{G})$ baseline to $(\mathbf{H})$ Month 8 .

Abbreviations: HEX, hard exudates; NPDR, nonproliferative diabetic retinopathy.

Table I Observations of the MERIT study at baseline and final follow-up

\begin{tabular}{llll}
\hline Characteristics & $\begin{array}{l}\text { At baseline - } \\
\text { mean }( \pm \text { SD) }\end{array}$ & $\begin{array}{l}\text { At } 8 \text { months }- \\
\text { mean }( \pm \text { SD })\end{array}$ & $p$-value \\
\hline BCVA in ETDRS letters & $50.93( \pm 16.654)$ & $50.6( \pm 18.95)$ & 0.959 \\
Contrast sensitivity $(\log M A R)$ & $1.15( \pm 0.39)$ & $0.97( \pm 0.43)$ & 0.31 \\
Central macular thickness $(\mu \mathrm{m})$ & $488.67( \pm 240.66)$ & $326.93( \pm 135.84)$ & 0.03 \\
Hard exudates area $\left(\mathrm{mm}^{2}\right)$ & $1.525( \pm 1.46)$ & $0.89( \pm 1.06)$ & 0.19 \\
Average threshold retinal sensitivity $(\mathrm{dB})$ & $6.99( \pm 8.896)$ & $11.91( \pm 7.64)$ & 0.29 \\
\hline
\end{tabular}

Abbreviations: BCVA, best-corrected visual acuity; ETDRS, Early Treatment Diabetic Retinopathy Study; logMAR, logarithm of the minimum angle of resolution; MERIT, macular edema resolution assessment with implantable dexamethasone in diabetic retinopathy. 
and steroids; ${ }^{11,13,18}$ however, in these cited studies, the quantification of HEX and assessment of RS as functional outcome were not carried out.

In our study, we noted a significant decrease in the CMT despite the short duration of follow-up; this demonstrates the efficacy of the dexamethasone implant in treating DME, as has been documented earlier. ${ }^{13,18}$ However, we did not notice a significant change in the BCVA, LCVA, and RS. This could be explained by the neuronal and structural damages secondary to the chronic nature of the DME, cataract progression, and presence of subfoveal HEX.

Corticosteroids reduce the inflammation and lead to recovery of blood-retina barrier, in addition to their antiVEGF property. Triamcinolone has been shown to cause resorption of HEX in various small case series in eyes with DME. ${ }^{3,5,6}$ Dexamethasone increases the vascular resistance and reduces permeability, which contributes to faster resolution of HEX.

Khairallah et $\mathrm{al}^{6}$ report resolution of HEX after single intravitreal triamcinolone injection at a mean of 8 months of follow-up. Similarly, Avci and Kaderli ${ }^{3}$ and Ciardella et $\mathrm{al}^{5}$ also report resolution of HEX in chronic DME with improvement in visual acuity. These studies have reported an increase in IOP in $25 \%-40 \%$ of eyes, which required antiglaucoma medications. None of these studies have evaluated functional outcomes. Moreover, dexamethasone implants have less frequent and transient episodes of increased IOP, which can be managed successfully with topical medications. ${ }^{19}$

Contrast sensitivity and RS are considered to be complementary tools in functional vision evaluation in DME. ${ }^{20}$ Vujosevic et $\mathrm{al}^{21}$ have previously found macular microperimetry to be an excellent additional tool to add to BCVA and CMT as a factor explaining visual function in patients with DME; they had also demonstrated a significant change in microperimetry results in patients with normalizing macular thickness in center-involving DME. Mastropasqua et $\mathrm{al}^{22}$ have found that RS, as measured by microperimetry, improved after dexamethasone implantation as early as 1-4 months after the injection. Similar findings have also been noted earlier by Querques et al. ${ }^{23}$ Our study demonstrated an improvement in functional outcome (RS) following dexamethasone implantation, even in the presence of subfoveal exudates.

Limitations of our study include the small sample size and lack of a control group. We did not evaluate SD-OCT structural changes with various outcome measures. We are unable to comment on comparative outcomes with antiVEGF therapy. We did not evaluate the distance of HEX from the fovea, which could be an important parameter to assess the foveal migration during follow-up.
The strength of this study was that we evaluated HEX resolution as the primary outcome measure in a prospective manner, along with the impact on the anatomical, visual, and functional changes. We performed a quantitative assessment of HEX using a validated semiautomated algorithm, not subjectively as done in earlier studies. Our objective approach could be used in the consideration of HEX as one of the outcome measures in clinical trials on DME evaluating various treatment modalities.

\section{Conclusion}

Our pilot study demonstrated reduction of total HEX and improvement in RS, along with a reduction in CMT. Poor visual outcomes were related to the persistence of subfoveal HEX; therefore, early consideration of dexamethasone implantation in eyes with HEX close to the fovea may be beneficial. Long-term studies with a larger sample size with a control group are warranted to establish management protocols for DME with significant subfoveal HEX.

\section{Acknowledgment}

The manuscript has not been presented at any meetings and no funding was provided for the study.

\section{Disclosure}

The authors report no conflicts of interest in this work.

\section{References}

1. Singh A, Stewart JM. Pathophysiology of diabetic macular edema. Int Ophthalmol Clin. 2009;49(2):1-11.

2. Yang CM. Surgical treatment for severe diabetic macular edema with massive hard exudates. Retina. 2000;20(2):121-125.

3. Avci R, Kaderli B. Intravitreal triamcinolone injection for chronic diabetic macular oedema with severe hard exudates. Graefes Arch Clin Exp Ophthalmol. 2006;244(1):28-35.

4. Avci R, Kaderli B, Akalp FD. Intravitreal triamcinolone injection for chronic diffuse diabetic macular oedema. Clin Exp Ophthalmol. 2006; 34(1):27-32.

5. Ciardella AP, Klancnik J, Schiff W, Barile G, Langton K, Chang S. Intravitreal triamcinolone for the treatment of refractory diabetic macular oedema with hard exudates: an optical coherence tomography study. $\mathrm{Br}$ J Ophthalmol. 2004;88(9):1131-1136.

6. Khairallah M, Zeghidi H, Ladjimi A, et al. Primary intravitreal triamcinolone acetonide for diabetic massive macular hard exudates. Retina. 2005;25(7):835-839.

7. Takagi H, Otani A, Kiryu J, Ogura Y. New surgical approach for removing massive foveal hard exudates in diabetic macular edema. Ophthalmology. 1999;106(2):249-256; discussion 256-247.

8. Nguyen QD, Brown DM, Marcus DM, et al. Ranibizumab for diabetic macular edema: results from 2 phase III randomized trials: RISE and RIDE. Ophthalmology. 2012;119(4):789-801.

9. Pemp B, Deak G, Prager S, et al; Diabetic Retinopathy Research Group Vienna. Distribution of intraretinal exudates in diabetic macular edema during anti-vascular endothelial growth factor therapy observed by spectral domain optical coherence tomography and fundus photography. Retina. 2014;34(12):2407-2415. 
10. Domalpally A, Ip MS, Ehrlich JS. Effects of intravitreal ranibizumab on retinal hard exudate in diabetic macular edema: findings from the RIDE and RISE phase III clinical trials. Ophthalmology. 2015;122(4): 779-786.

11. Larsson J, Kifley A, Zhu M, et al. Rapid reduction of hard exudates in eyes with diabetic retinopathy after intravitreal triamcinolone: data from a randomized, placebo-controlled, clinical trial. Acta Ophthalmol. 2009;87(3):275-280

12. Sutter FK, Simpson JM, Gillies MC. Intravitreal triamcinolone for diabetic macular edema that persists after laser treatment: three-month efficacy and safety results of a prospective, randomized, double-masked, placebo-controlled clinical trial. Ophthalmology. 2004;111(11): 2044-2049.

13. Mehta H, Fraser-Bell S, Yeung A, et al. Efficacy of dexamethasone versus bevacizumab on regression of hard exudates in diabetic maculopathy: data from the BEVORDEX randomised clinical trial. Br J Ophthalmol. 2015;100(7):1000-1004.

14. Diabetic Retinopathy Clinical Research Network, Wells JA, Glassman AR, et al. Aflibercept, bevacizumab, or ranibizumab for diabetic macular edema. N Engl J Med. 2015;372(13):1193-1203.

15. Gilbert EH, Lowenstein SR, Koziol-McLain J, Barta DC, Steiner J. Chart reviews in emergency medicine research: where are the methods? Ann Emerg Med. 1996;27(3):305-308.

16. Emery JM. Kelman phacoemulsification, patient selection. In: Emery JM, MoIntyre DJ, editors. Extracapsular cataract surgery: St. Louis, MO: CV Mosby; 1983:95-100.
17. Gonzalez RC, Woods RE. Digital Image Processing. Upper Saddle River, NJ: Prentice Hall; 2008.

18. Scaramuzzi M, Querques G, Spina CL, Lattanzio R, Bandello F. Repeated intravitreal dexamethasone implant (Ozurdex) for diabetic macular edema. Retina. 2015;35(6):1216-1222.

19. Malcles A, Dot C, Voirin N, et al. Safety of intravitreal dexamethasone implant (Ozurdex): the safodex study. Incidence and risk factors of ocular hypertension. Retina. 2016;37(7):1352-1359.

20. Kim YH, Yun C, Kim JT, Kim SW, Oh J, Huh K. The correlation between retinal sensitivity assessed by microperimetry and contrast sensitivity in diabetic macular oedema. Br J Ophthalmol. 2014;98(12):1618-1624.

21. Vujosevic S, Midena E, Pilotto E, Radin PP, Chiesa L, Cavarzeran F. Diabetic macular edema: correlation between microperimetry and optical coherence tomography findings. Invest Ophthalmol Vis Sci. 2006; 47(7):3044-3051.

22. Mastropasqua R, Toto L, Borrelli E, et al. Morphology and function over a one-year follow up period after intravitreal dexamethasone implant (Ozurdex) in patients with diabetic macular edema. PLoS One. 2015;10(12):e0145663.

23. Querques G, Lattanzio R, Querques L, et al. Impact of intravitreal dexamethasone implant (Ozurdex) on macular morphology and function. Retina. 2014;34(2):330-341.
Clinical Ophthalmology

\section{Publish your work in this journal}

Clinical Ophthalmology is an international, peer-reviewed journa covering all subspecialties within ophthalmology. Key topics include: Optometry; Visual science; Pharmacology and drug therapy in eye diseases; Basic Sciences; Primary and Secondary eye care; Patien Safety and Quality of Care Improvements. This journal is indexed on

Submit your manuscript here: http://www.dovepress.com/clinical-ophthalmology-journal

\section{Dovepress}

PubMed Central and CAS, and is the official journal of The Society of Clinical Ophthalmology (SCO). The manuscript management system is completely online and includes a very quick and fair peer-review system, which is all easy to use. Visit http://www.dovepress.com/ testimonials.php to read real quotes from published authors. 